\title{
Stent Thrombosis Secondary to Ticagrelor Resistance as Demonstrated by Thromboelastography
}

\begin{abstract}
Introduction: We describe a case of stent thrombosis while on ticagrelor, with demonstrated ticagrelor resistance on Thromboelastography Platelet Mapping (TEG). Case description: The patient is a 61 year old male with a ST elevation myocardial infarction (STEMI) requiring stents 4 days before admission. The patient returned with a stent thrombosis, due to ticagrelor noncompliance. During his stay, the patient was restarted on ticagrelor, with all doses documented to have been given as scheduled. He had a new STEMI 2 days later in the hospital and was sent again for PCI, which showed another stent thrombosis. Thrombelastography (B) Platelet Mapping ${ }^{\mathrm{TM}}$ showed resistance to ticagrelor with $7.0 \%$ inhibition and a maximum amplitude (MA) of $71.2 \mathrm{~mm}$ with ADP and was replaced by prasugrel. TEG on prasugrel showed inadequate inhibition, with an inhibition of $0.0 \%$ with a MA of 77.8 with ADP, but retesting the sample with whole blood luminescence aggregometry showed adequate inhibition with ADP. The patient was discharged on prasugrel and had no further episodes of stent thrombosis to date.

Discussion: Stent thrombosis is a dreaded complication of on-treatment platelet reactivity. With ticagrelor, stent thrombosis is rare due to its nature as a direct P2Y12 inhibitor that does not require metabolization. There is only one other reported case, to our knowledge, of stent thrombosis with laboratory confirmed ticagrelor resistance. An analysis of the Platelet inhibition and patient Outcomes trial showed that stent thrombosis is less frequent with ticagrelor compared to clopidogrel. There is little research to explain ticagrelor resistance and known drug interactions are not clinically significant. Testing for P2Y12 inhibitor resistance is an area in need of elucidation, with few consensus guidelines regarding the definition of resistance and how the multiple platelet function tests compare to one another.
\end{abstract}

Keywords: Stent thrombosis; Ticagrelor; Prasugrel; P2Y12 Inhibitor; Thromboelastography; Whole Blood Luminescence Aggregometry; Whole Blood Impedance Aggregometry; Acute coronary syndrome; Ticagrelor resistance

\author{
Case Report \\ Volume 6 Issue 3 - 2016 \\ Addi Suleiman ${ }^{1}$, Ahsan Khan ${ }^{2 *}$, Sahitya \\ Posimreddy ${ }^{2}$, Claudia Komer ${ }^{3}$ and Abbas \\ Shehadeh ${ }^{1}$ \\ ${ }^{1}$ Department of Cardiology, New York Medical College/Saint \\ Michael's Medical Center, USA \\ ${ }^{2}$ Department of Internal Medicine, New York Medical College/ \\ Saint Michael's Medical Center, USA \\ ${ }^{3}$ Department of Anesthesia, New York Medical College/Saint \\ Michael's Medical Center, USA \\ *Corresponding author: Ahsan Khan, Department of \\ Internal Medicine, New York Medical College/Saint Michael's \\ Medical Center, 111 Central Ave, Newark, NJ - 07102, USA, \\ Tel: 973-508-4690; \\ Email: Akhan07@gmail.com
}

Received: July 31, 2016 | Published: August 02, 2016

\section{Introduction}

Stent thrombosis is a dreaded complication of percutaneous coronary interventions (PCI). It is a prominent cause of mortality and major bleeding [1]. Antiplatelet therapy has played an important role in mitigating this adverse event, yet it is still a major complication. Clopidogrel, an irreversible P2Y12 inhibitor has been a mainstay of antiplatelet therapy for years. Clopidogrel's nature as a prodrug means that certain patients have unsatisfactorily inhibited platelet activity due to differences in drug metabolism. This lead to the production of a new generation of P2Y12 inhibitors, most famously ticagrelor and prasugrel, of which ticagrelor provides platelet inhibition without requiring metabolism. This results in more potent and consistent platelet inhibition. Prasugrel is infrequently used as a first-line treatment for acute coronary syndromes (ACS), due to its increased risk of bleeding. Ticagrelor, on the other hand is used more commonly, because of its lower rates of cardiovascular events, cardiovascular death and all cause mortality compared to clopidogrel, while being comparable in its adverse event profile [2]. Stent thrombosis has been shown to be less frequent with ticagrelor than clopidogrel [1]. In fact, stent thrombosis while on ticagrelor is a rare occurrence. To our knowledge, there is only one reported case regarding stent thrombosis in a patient actively treated with ticagrelor [3]. There are numerous platelet function tests (PFT) now available to assess for P2Y12 inhibitor resistance, although guidelines for their use is not clear. We will be presenting a case of stent thrombosis while on treatment with ticagrelor, with resistance demonstrate by thromboelastogram.

\section{Case}

The patient is a 61 year old male with a past medical history of coronary artery disease, hypertension, diabetes and HIV. He had an ST elevation myocardial infarction only 4 days before the day of admission. At that time, he had a percutaneous coronary intervention (PCI), which showed $100 \%$ stenosis of right coronary artery (RCA) for which a drug eluting stent was placed, as well as $85 \%$ stenosis in the proximal left anterior descending artery (LAD) for which two stents were placed. The patient was given ticagrelor during the admission, but was unable to continue it after discharge, due to problems with insurance coverage. The 
patient presented again to the hospital with typical chest pain and was determined to have another ST elevation myocardial infarction. The patient immediately went for PCI, which showed acute distal LAD and RCA stent thrombosis and 90\% stenosis of the 1st diagonal branch of the LAD (D1). The proximal LAD stent was still patent. Pronto (Vascular Solutions, Inc., Minneapolis, Minnesota) thrombus extraction catheter was used in the LAD, followed by dilation with a Quantum (Boston Scientific Corporation, Marlborough, MA) balloon catheter. The patient was continued on dual antiplatelet therapy with ticagrelor with every dose documented to have been administered by hospital staff. The patient again had chest pain 2 days later with new ST elevations in V5 and V6. The patient underwent PCI and had 100\% stenosis of D1 due to subacute stent thrombosis. Angioplasty of D1 was done, followed by thrombectomy with a Pronto catheter, followed by kissing balloon angioplasty at the LAD and D1 bifurcation, after which ticagrelor was restarted. Eptifibatide drip was initially administered, but was soon discontinued due to an allergic reaction. Thrombelastography ${ }^{\circledR}$ Platelet Mapping ${ }^{\mathrm{TM}} \quad$ (TEG) (Haemoscope Corporation, Niles, IL, USA) showed resistance to ticagrelor with $7.0 \%$ inhibition and a maximum amplitude (MA) of $71.2 \mathrm{~mm}$ with ADP. Prasugrel was started in its place, but a repeat TEG on prasugrel still showed resistance, with an inhibition of $0.0 \%$ with a MA of 77.8 with ADP. Whole blood luminescence aggregometry (WBLA) (Chrono-Log Corporation, Columbia, MD, USA) showed appropriate inhibition with an aggregate reading of $4 \mathrm{ohms}$. The patient was finally discharged on an antiplatelet regimen of aspirin and prasugrel.

\section{Discussion}

Stent thrombosis, while on treatment with ticagrelor is rare. There is only one reported case, to our knowledge, of such an event, which was reported by Musallam et al. [3]. The PLATelet inhibition and patient Outcomes (PLATO) trial involved 18,624 patients hospitalized for ACS, with 11,289 (61\%) having at least one intracoronary stent. An analysis of this trial showed that the rate of possible, probable and definite stent thrombosis in patients on Ticagrelor was $2.94 \%(n=154)$. Stent thrombosis in patients taking clopidogrel was higher at 3.77\% [ $(\mathrm{n}=201)$ : HR $0.77,95 \%$ CI $(0.62-0.95)]$. The rate for definite stent thrombosis was $1.37 \%(n=71)$, which was similarly lower than the rate of definite stent thrombosis on clopidogrel 1.93\% ( $n=105)$; (HR 0.67, 95\% confidence interval [CI] (0.50 - 0.90). This was consistent across a variety of variables, including type of stent used, CYP2C19 genetic status and use of other antiplatelet agents. An interesting finding was that the reduction of stent thrombosis was greater with ticagrelor compared to clopidogrel for late $(>30$ days) and subacute ( $24 \mathrm{~h}-30$ days), but no difference was seen for acute stent thrombosis $(<24 \mathrm{~h})$. This may be, because ticagrelor achieves adequate platelet inhibition in 2 hours after the loading dose in half of patients with a STEMI and 4 hours in remaining patients [4]. Contrastingly, the cases mentioned here and the case reported by Musallam et al. [3] were subacute stent thrombosis [3].

A significant amount of research has been done to explain high on-treatment platelet reactivity for clopidogrel, including CYP2C19*2 polymorphism, inflammation and drug interactions
[2]. In comparison, there is little information explaining possible causes of ticagrelor resistance. Zhou et al determined that CYP3A4 and CYP3A5 are predominantly responsible for the metabolism of ticagrelor into its active and inactive metabolites, but this is of little significance since ticagrelor achieves sufficient efficacy before being metabolized [2]. The IMPRESSION trial demonstrated retarded and reduced effect of ticagrelor in patients coadministered with morphine in comparison to ticagrelor with placebo. There was one stent thrombosis in the morphine arm compared to none in the placebo arm, but this was not a statistically significant difference [5]. Similarly, smoking resumption after brief cessation after STEMI was associated with a modest increase in platelet reactivity, although unlikely to the extent that it would have clinical implications [6]. Other factors independent of P2Y12 inhibition may contribute to high platelet reactivity, including catecholamine mediated platelet activation, P2Y1 dependent platelet aggregation and up regulation of other P2Y12 independent pathways [7].

An interesting aspect of this case was the use of TEG and WBLA to assess for P2Y12 inhibitor resistance. TEG is a viscoelastic hemostatic assay which measures the physical properties of clot formation under low shear stress. Whole blood is rotated in a cup to simulate sluggish venous flow and clot formation, while data is recorded by a pin suspended in the sample and connected to an electrical transducer. The data is translated into numerical values and graphs, which are used to interpret the contribution of blood components in clot formation. The MA represents clot strength and is the measurement primarily used to assess platelet activity $[8,9]$. WBLA is another PFT which measures the resistance between two electrodes immersed in whole blood as platelets adhere to them and the clot forms. WBLA has this function in common with whole blood impedance aggregometry, but adds a function for measuring ATP secretion $[9,10]$. These modalities can test the effectiveness of aspirin and P2Y12 inhibitors by activating platelets with arachidonic acid and ADP, respectively and measuring the level of inhibition from these agents [11].

High on treatment platelet reactivity, as measured by many PFT, is associated with increased adverse clinical events. Still, there is a paucity of guidelines for what constitutes antiplatelet resistance. Some consensus guidelines for P2Y12 inhibitor resistance have been offered for some PFT, yet the authors admit that adjusting management by any of the cut-off values has not been shown to improve clinical outcomes [11]. Many studies use a standard of $10 \%$ decrease in platelet aggregation after P2Y12 inhibitor initiation, but this cannot be done on a patient already on treatment [12]. Bochsen et al. [12] performed TEG in 43 healthy blood donors who were not taking any platelet inhibiting agents. Their ADP receptor inhibition ranged from $0 \%$ to $58 \%$ [13]. In a study by Collyer et al. [13], the ADP inhibition range for for a control group of preoperative patients not on antiplatelet agents was much broader at $23-92 \%$ [14]. A study found that a MA with ADP of $>47 \mathrm{~mm}$ was a strong predictor for longerterm ischemic events following coronary stenting, suggesting on treatment platelet reactivity as a possible cause [15]. A study by Bliden et al set the definition of on treatment platelet reactivity with clopidogrel arbitrarily at 70\% ADP induced aggregation, but by this standard found that patients who exhibited high 
platelet reactivity on clopidogrel via TEG before an elective stent were at higher risk for postprocedure ischemic events [16]. The manufacturer guidelines are that a percent inhibition $>50 \%$ inhibition indicate an increased risk of bleeding, but do not comment on cutoff for antiplatelet resistance. Boris et al. determined their cut off for clopidogrel nonresponse at $5 \mathrm{ohms}$ by subtracting 3 standard deviations from the mean 6 minute impedance from ADP of healthy clopidogrel-naive participants. The normal values at our laboratory for WBLA is 9-25 ohms. Multiple PFT on the same sample can yield highly variable results and there is a lack of standardization between labs $[9,15]$.

This case raises many interesting and important questions regarding the management of ticagrelor resistance. Prasugrel seems to have been successful in this patient. He has presented to the hospital several times since, but for reasons unrelated to acute coronary syndromes and had no further episodes of stent thrombosis. Is WBLA a more reliable test than TEG, or are the results only a matter of variance between different modalities? What should be done when two PFT have different results, especially in light of the lack of data showing superiority of one over the other? Should routine testing for platelet reactivity be performed once an antiplatelet agent is used to replace the other due to resistance? Is there a mechanism by which prasugrel inhibits platelet aggregation that ticagrelor does not? Hopefully, these questions will be answered in future research.

\section{References}

1. Steg PG, Harrington RA, Emanuelsson H, Katus HA, Mahaffey KW, et al. (2013) Stent Thrombosis With Ticagrelor Versus Clopidogrel in Patients With Acute Coronary Syndromes: An Analysis From the Prospective, Randomized PLATO Trial. Circulation 128(10): 10551065.

2. Abergel E, Nikolsky E (2010) Ticagrelor: An investigational oral antiplatelet treatment for reduction of major adverse cardiac events in patients with acute coronary syndrome. Vasc Health Risk Manag 6: 963-977.

3. Musallam A, Lev EI, Roguin A (2014) Stent thrombosis in a patient with high on-treatment platelet reactivity despite ticagrelor treatment. Eur Heart J Acute Cardiovasc Care 4(1): 85-87.

4. Zhou D, Andersson TB, Grimm SW (2010) In Vitro Evaluation of Potential Drug-Drug Interactions with Ticagrelor: Cytochrome P450 Reaction Phenotyping, Inhibition, Induction, and Differential Kinetics. Drug Metab Dispos 39(4): 703-710.
5. Kubica J, Adamski P, Ostrowska M, Sikora J4, Kubica JM, et al. (2015) Morphine delays and attenuates ticagrelor exposure and action in patients with myocardial infarction: the randomized, double-blind, placebo-controlled IMPRESSION trial. Eur Heart J 37(3): 245-252.

6. Patti G, Polacco M, Taurino E, Gaudio C, Greco C (2016) Effects of cigarette smoking on platelet reactivity during P2Y12 inhibition in patients with myocardial infarction undergoing drug-eluting stent implantation: results from the prospective cigarette smoking on platelet reactivity (COPTER) study. J Thromb Thrombolysis 41(4): 648-653.

7. Nguyen TA, Diodati JG, Pharand C (2005) Resistance to clopidogrel: A review of the evidence. J Am Coll Cardiol 45(8): 1157-1164.

8. TEG Platelet Mapping Assay. Haemonetics.

9. Quellec SL, Bordet J-C, Negrier C, Dargaud Y (2016) Comparison of current platelet functional tests for the assessment of aspirin and clopidogrel response. Thromb Haemost 116(4).

10. Chrono-log whole blood and optical platelet aggregometers.

11. Gurbel PA, Becker RC, Mann KG, Steinhubl SR, Michelson AD (2007) Platelet Function Monitoring in Patients With Coronary Artery Disease. J Am Coll Cardiol 50(19): 1822-1834.

12. Bochsen L, Wiinberg B, Kjelgaard-Hansen M, Steinbruchel DA, Johansson PI (2007) Evaluation of the TEG $®$ Platelet Mapping ${ }^{\mathrm{TM}}$ assay in blood donors. Thromb J 5: 3.

13. B Collyer TC, Gray DJ, Sandhu R, Berridge J, Lyons G (2009) Assessment of platelet inhibition secondary to clopidogrel and aspirin therapy in preoperative acute surgical patients measured by Thrombelastography(R) Platelet Mapping TM. Br J Anaesth 102(4): 492-498.

14. Gurbel PA, Bliden KP, Navickas IA, Mahla E, Dichiara J, et al. (2010) Adenosine diphosphate-induced platelet-fibrin clot strength: A new thrombelastographic indicator of long-term poststenting ischemic events. Am Heart J 160(2): 346-354.

15. Bouman HJ, Parlak E, van Werkum JW, Breet NJ, ten Cate H, et al. (2010) Which platelet function test is suitable to monitor clopidogrel responsiveness? A pharmacokinetic analysis on the active metabolite of clopidogrel. J Thromb Haemost 8(3): 482-488.

16. Ivandic BT, Schlick P, Staritz P, Kurz K, Katus HA (2006) Determination of Clopidogrel Resistance by Whole Blood Platelet Aggregometry and Inhibitors of the P2Y12 Receptor. Clin Chem 52(3): 383-388. 